\title{
Correlation Test by Reduced-Spread of Fuzzy Variance
}

\author{
Man Ki Kang ${ }^{1, a}$ \\ ${ }^{a}$ Department of Data Information Science, Dong-eui University
}

\begin{abstract}
We propose some properties for a fuzzy correlation test by reduced-spread fuzzy variance for sample fuzzy data. First, we define the condition of fuzzy data for repeatedly observed data or that which includes error term data. By using the average of spreads for fuzzy numbers, we reduce the spread of fuzzy variance and define the agreement index for the degree of acceptance and rejection. Given a non-normal random fuzzy sample, we have bivariate normal distribution by apply Box-Cox power fuzzy transformation and test the fuzzy correlation for independence between the variables provided by the agreement index.
\end{abstract}

Keywords: Reduced-spread fuzzy variance, degree of acceptance and rejection, fuzzy significance probability, agreement index, Box-Cox power fuzzy transformation.

\section{Introduction}

In many real situations, uncertainty data comes from randomness and fuzziness. Randomness models the stochastic variability of all possible outcomes for an experiment and fuzziness that describes the vagueness of a given outcome.

For the uncertainty data, a fuzzy hypothesis testing method that is related to the fuzzy expected value (combined with other fuzzifications) have shown to be very useful inferences on distributions of real-valued random variables by Gizegorzewski (2000).

Watanabe and Imaizumi (1993) considered the fuzzy hypothesis by a constructed set $\left\{\left(H_{0}(\psi)\right.\right.$, $\left.\left.H_{1}(\psi)\right) \mid \psi \in \Theta\right\}$ but we have the fuzzy negation of the assertion is taken to be the fuzzy null hypothesis $H_{f 0}$ and the fuzzy assertion itself is taken to be the fuzzy alternative hypothesis $H_{f 1}$.

Kang and Seo (2009) defined the fuzzy hypotheses membership function. They consider the fuzzy hypothesis as

$$
H_{f 0}: m_{\theta}=m_{\theta_{0}} \quad \text { or } \quad H_{f 0}: m_{\theta}<m_{\theta_{0}}, \quad \theta \in \Omega,
$$

where $\Omega$ is parameter space. Kang et al. (2003) defined an agreement index by area ratio by a fuzzy hypotheses membership function with respect to membership function of the fuzzy critical region, and obtained the results by the grade for the judgement of acceptance or rejection for the fuzzy hypotheses.

We suggest some properties for a fuzzy hypothesis test using fuzzy significance probability by an agreement index with a reduced-spread fuzzy variance and covariance by an average of fuzzy number center and spreads.

This paper is organized as follows. First, we define the class of fuzzy sets and the condition of fuzzy data for repeatedly observed data or that which includes error term data in a random experiment. In Section 3, we suggest a reduce-spread fuzzy variance and covariance by the average of the

\footnotetext{
This work was supported by the Dong-eui University Grant(2011AA091).

${ }^{1}$ Professor, Department of Data Information Science, Dong-eui University, 995 Eomgwangno, Busanjin-gu, Busan 614-

714, Korea. E-mail: mkkang@deu.ac.kr
} 
fuzzy number center and spreads. In Section 4, we suggest an agreement index by the area ratio for the acceptance or rejection degree of fuzzy hypotheses. In Section 5, we show a power-normal distribution by Box-Cox power fuzzy transformation and fuzzy $t$-test statistics to test the independence of bivariate normal distribution. Finally, in Section 6, we illustrate the fuzzy hypothesis test with a fuzzy significance probability by an agreement index for a non-normal data of blood pressure samples and infer a conclusion.

\section{Preliminaries}

Let $K_{c}\left(\mathfrak{R}^{p}\right)$ be the class of the non-empty compact convex subsets of $\mathfrak{R}^{p}$. We will consider the class of fuzzy sets

$$
F_{c}\left(\mathfrak{R}^{p}\right)=\left\{U: \mathfrak{R}^{p} \rightarrow[0,1] \mid U_{\delta} \in K_{c}\left(\mathfrak{R}^{p}\right) \text { for all } \delta \in[0,1]\right\},
$$

where $U_{\delta}$ stands for the $\delta$-level of $U$ (i.e. $U_{\delta}=\left\{x \in \mathfrak{R}^{p} \mid U(x) \geq \delta\right\}$ ) for all $\delta \in(0,1], \delta$ is the precision of data in statistical concept and $U_{0}$ is the closure of the support of $U$ by Colubi (2009).

An example of fuzzy set on $F_{c}(\mathfrak{R})$ is considered as the called triangular fuzzy number $A=$ $\left[A_{l}, A_{c}, A_{r}\right]$ with the center $A_{c} \in \mathfrak{R}$ and the "spreads" $A_{l}, A_{r} \in \mathfrak{R}$. We have fuzzy number so called $\mathrm{LR}$ (Left, Right)-fuzzy numbers $A:=[l, c, r]_{L R}$ with modal value $c \in \mathfrak{R}$, left and right spreads $l, r \in \mathfrak{R}$ decreasing left and right shape functions.

Thus, we have fuzzy membership function $A$ as

$$
m_{A}(x)=\left\{\begin{aligned}
\frac{x-l}{c-l}, & \text { if } x \leq c, \\
-\frac{x-r}{r-l}, & \text { if } x>c .
\end{aligned}\right.
$$

To satisfy the statistical fuzzy data, a fuzzy number $A$ in $\mathfrak{R}$ is said to be convex if for any real numbers $x, y, z \in \mathfrak{R}$ with $x \leq y \leq z$,

$$
m_{A}(y) \geq m_{A}(x) \wedge m_{A}(z)
$$

with $\wedge$ standing for minimum.

In addition, statistical fuzzy data $A$ is called normal if the following holds

$$
\bigvee_{x} m_{A}(x)=1
$$

A $\delta$-level set of a fuzzy number data $A$ is a set of $[A]^{\delta}$ and defined by

$$
[A]^{(\delta)}=\left\{x \mid m_{A}(x) \geq \delta, 0 \leq \delta \leq 1\right\} .
$$

A $\delta$-level set of fuzzy number data $A$ is a convex fuzzy set, which is a closed and bounded interval denoted by $[A]^{(\delta)}=\left[A_{l}, A_{c}, A_{r}\right]^{(\delta)}$.

The space $F_{c}\left(\mathfrak{R}^{p}\right)$ can be endowed with the sum and the product a scalar based on Zadeh's extension principle. Let $A$ and $B$ be fuzzy numbers data in $\mathfrak{R}$ and let $\odot$ be a binary operation defined in $\mathfrak{R}$. Then the operation $\odot$ can be extended to the fuzzy numbers $A$ and $B$ by defining the relation of extension principle as; given $A, B, \forall x, y, z \in \mathfrak{R}$;

$$
m_{A \odot B}(z)=\bigvee_{z=x \odot y}\left(m_{A}(x) \wedge m_{B}(y)\right) .
$$




\section{Reduced-Spread of Fuzzy Variance}

Let $(\Omega, A, P)$ be the probability space. A random fuzzy variable(RFV) is a mapping $\chi: \Omega \rightarrow F_{c}\left(\Re^{p}\right)$ so that the $\delta$-level functions $\chi^{(\delta)}: \Omega \rightarrow K_{c}\left(\mathfrak{R}^{p}\right)$, defined so that $\chi^{(\delta)}(\theta)=(\chi(\theta))^{(\delta)}$ for all $\theta \in \Omega$, are the random sets.

Definition 1. If $\chi: \Omega \rightarrow F_{c}\left(\mathfrak{R}^{p}\right)$ is a RFV such that $E\left(\sup _{x \in \chi_{0}}\|x\|\right)<\infty$ with $\left|\chi_{0}\right|(\omega)=\sup \left\{|x| \mid x \in \chi_{0}\right\}$ for all $\omega \in \Omega$, then the expected fuzzy value (or mean) of $\chi$ is the unique, that is,

$$
(\tilde{E}(\chi))^{(\delta)}=\left\{E(X \mid P) \mid X: \Omega \rightarrow \mathfrak{R}^{p}, X \in L^{1}(\Omega, A, P), X \in \chi^{(\delta)} \text { a.s. }[P]\right\},
$$

where $L_{1}$ is a metrics for left, center and right spreads form origin " 0 " of fuzzy number data, respectively.

Definition 2. Given a probability space $(\Omega, A, P)$, if $R F V$ have $\chi$ then $E(|\chi|)<\infty$ fuzzy variance of $\chi$ is defined as

$$
\sigma_{\chi}^{2}=\operatorname{Var}(\chi)=E\left(\left[D_{A}(\chi, \tilde{E}(\chi))\right]^{2}\right),
$$

where $D_{A}^{2}$ are $\left(c^{2}+\min \left\{l^{2}, r^{2}\right\}\right) / 2, c^{2}$ and $\left(c^{2}+\max \left\{l^{2}, r^{2}\right\}\right) / 2$ for $\chi \ominus \tilde{E}(\chi)=[l, c, r]$ by Equation (2.6).

As a precise sample $\left(\boldsymbol{x}_{\mathbf{1}}, \boldsymbol{x}_{\mathbf{2}}\right)^{\prime}$ of $n$ precise realizations $x_{1 i}, x_{2 i} \in \mathfrak{R}$ may be regarded as vector in $\mathfrak{R}^{n}$, each random sample $x_{1 i}, x_{2 i}$ have a sample $\left(\boldsymbol{x}_{\mathbf{1}}, \boldsymbol{x}_{\mathbf{2}}\right)^{\prime}$ of $n$ fuzzy realizations $\chi_{1 i}, \chi_{2 i} \in F(\mathfrak{R})$, $i=1,2, \ldots, n$, may be regarded as a fuzzy vector by Equation (2.1). A modeling the fuzziness of data were described the fuzziness of a fuzzy sample $\boldsymbol{\chi}=\left(\chi_{1}, \chi_{2}\right)^{\prime}=\left\{\left(\chi_{11}, \chi_{21}\right)^{\prime},\left(\chi_{12}, \chi_{22}\right)^{\prime}, \ldots,\left(\chi_{1 n}, \chi_{2 n}\right)^{\prime}\right\}$.

If we observe an object at three times by minimum, median and maximum or including error term data, then we organize a fuzzy number to $\chi_{k i}=\left[\chi_{\min _{k i}}, \chi_{\operatorname{med}_{k i},} \chi_{\max _{k i}}\right], k=1,2, i=1,2, \ldots, n$.

Thus, we have the fuzzy sample mean as

$$
\begin{aligned}
{\left[\overline{\chi_{k}}\right]^{(\delta)} } & =\left[\frac{1}{n} \sum_{i=1}^{n} \chi_{k i}\right]^{(\delta)} \\
& =\left[\frac{1}{n} \sum_{i=1}^{n} \chi_{\min _{k i}}, \frac{1}{n} \sum_{i=1}^{n} \chi_{\operatorname{med}_{k i}}, \frac{1}{n} \sum_{i=1}^{n} \chi_{\max _{k i}}\right]^{(\delta)} \\
& =\left[\overline{x_{k l}}, \overline{x_{k c}}, \overline{x_{k r}}\right]^{(\delta)},
\end{aligned}
$$

by Definition 1 for $\delta$-level, $k=1,2$.

For $\delta=0$, if we have $S_{k}^{2^{(\delta=0)}}=S_{k}^{2}$ then we make clear that the variation of an RFV around its variance value will be considered as

$$
\begin{aligned}
S_{k}^{2} & =\frac{1}{n-1} \sum_{i=1}^{n}\left(\chi_{k i} \ominus \overline{\chi_{k}}\right)^{2}=\frac{1}{n-1} \sum_{i=1}^{n}\left(\left[x_{k l i}, x_{k c i}, x_{k r i}\right] \ominus\left[\overline{x_{k l}}, \overline{x_{k c}}, \overline{x_{k r}}\right]\right)^{2} \\
& \neq \frac{1}{n-1} \sum_{i=1}^{n} \chi_{k i}^{2} \ominus \frac{n}{n-1}{\overline{\chi_{k}}}^{2}=\frac{1}{n-1}\left\{\sum_{i=1}^{n}\left[x_{k l i}^{2}, x_{k c i}^{2}, x_{k r i}^{2}\right] \ominus n\left[{\overline{x_{k l}}}^{2},{\overline{x_{k c}}}^{2},{\overline{x_{k r}}}^{2}\right]\right\}
\end{aligned}
$$

$k=1,2$ by fuzzy operation of Equation (2.6). 
The above spread of $S_{\chi_{k}}^{2}$ has too wide spread or negative value. Our opinion, $S_{\chi_{k}}^{2}$ is unsuitable fuzzy variance by Equation (3.4). If we let $\left[l_{k i}, l_{k i}, l_{k i}\right]<\chi_{k i} \ominus \overline{\chi_{k}}<\left[r_{k i}, r_{k i}, r_{k i}\right]$ for $\left[l_{k i}, c_{k i}, r_{k i}\right]$ then

$$
\begin{aligned}
{\left[\min \left\{l_{k i}^{2}, r_{k i}^{2}\right\}, \min \left\{l_{k i}^{2}, r_{k i}^{2}\right\}, \min \left\{l_{k i}^{2}, r_{k i}^{2}\right\}\right] } & <\left(\chi_{k i} \ominus \overline{\chi_{k}}\right)^{2} \\
& <\left[\max \left\{l_{k i}^{2}, r_{k i}^{2}\right\}, \max \left\{l_{k i}^{2}, r_{k i}^{2}\right\}, \max \left\{l_{k i}^{2}, r_{k i}^{2}\right\}\right],
\end{aligned}
$$

$k=1,2, i=1, \ldots, n$.

However, in case of overlapping $\chi_{k i}$ by $\overline{\chi_{k}}$, we have

$$
[0,0,0]<\left(\chi_{k i} \ominus \overline{\chi_{k}}\right)^{2}<\left[\max \left\{l_{k i}^{2}, r_{k i}^{2}\right\}, \max \left\{l_{k i}^{2}, r_{k i}^{2}\right\}, \max \left\{l_{k i}^{2}, r_{k i}^{2}\right\}\right], \quad k=1,2, i=1, \ldots, n .
$$

In order to reduce the spreads of fuzzy numbers for sample fuzzy variance, we have reducedspread fuzzy variance in this paper as

$$
\begin{aligned}
\widetilde{S_{k}^{2}} \doteq \frac{1}{n-1} \sum_{i=1}^{n}\left(\left[\frac{c_{k i}^{2}+\min \left\{l_{k i}^{2}, r_{k i}^{2}\right\}}{2}, c_{k i}^{2}, \frac{c_{k i}^{2}+\max \left\{l_{k i}^{2}, r_{k i}^{2}\right\}}{2}\right], \text { for } l_{k i}>0 \text { or } r_{k i}<0,\right. \\
\left.\quad\left[\frac{0+c_{k i}^{2}}{2}, c_{k i}^{2}, \frac{c_{k i}^{2}+\max \left\{l_{k i}^{2}, r_{k i}^{2}\right\}}{2}\right], \text { for } l_{k i} \leq 0 \text { and } r_{k i}>0\right) \\
=\left[S_{k l}^{2}, S_{k c}^{2}, S_{k r}^{2}\right], \quad k=1,2,
\end{aligned}
$$

where $\doteq$ is analogously equal by Definition 2 .

In case of fuzzy covariance, for $\delta=0$, we also make clear that

$$
\begin{aligned}
& \operatorname{Cov}\left(\chi_{1}, \chi_{2}\right) \\
& =\frac{1}{n-1} \sum_{i=1}^{n}\left(\chi_{1 i} \ominus \overline{\chi_{1}}\right) \cdot\left(\chi_{2 i} \ominus \overline{\chi_{2}}\right) \\
& =\frac{1}{n-1} \sum_{i=1}^{n}\left(\left[x_{1 l i}, x_{1 c i}, x_{1 r i}\right] \ominus\left[\overline{x_{1 l}}, \overline{x_{1 c}}, \overline{x_{1 r}}\right]\right) \otimes\left(\left[x_{2 l i}, x_{2 c i}, x_{2 r i}\right] \ominus\left[\overline{x_{2 l}}, \overline{x_{2 c}}, \overline{x_{2 r}}\right]\right) \\
& \neq \frac{1}{n-1}\left\{\sum_{i=1}^{n}\left[x_{1 l i}, x_{1 c i}, x_{1 r i}\right]\left[x_{2 l i}, x_{2 c i}, x_{2 r i}\right] \ominus n\left(\frac{1}{n} \sum_{i=1}^{n}\left[x_{1 l i}, x_{1 c i}, x_{1 r i}\right]\right)\left(\frac{1}{n} \sum_{i=1}^{n}\left[x_{2 l i}, x_{2 c i}, x_{2 r i}\right]\right)\right\}
\end{aligned}
$$

by fuzzy operation of Equation (2.6).

However, the spreads of fuzzy numbers for sample fuzzy covariance were very wide, and therefore we reduced the spreads for sample fuzzy covariance as;

$$
\begin{aligned}
& \operatorname{\operatorname {Cov}(\chi _{1},\chi _{2})} \\
& =\frac{1}{n-1} \sum_{i=1}^{n}\left(\left[x_{1 l i}, x_{1 c i}, x_{1 r i}\right] \ominus\left[\overline{x_{1 l}}, \overline{x_{1 c}}, \overline{x_{1 r}}\right]\right) \otimes\left(\left[x_{2 l i}, x_{2 c i}, x_{2 r i}\right] \ominus\left[\overline{x_{2 l}}, \overline{x_{2 c}}, \overline{x_{2 r}}\right]\right) \\
& \doteq \frac{1}{n-1} \sum_{i=1}^{n}\left(\left[\frac{\min \left\{l_{1 i} l_{2 i}, l_{1 i} r_{2 i}, l_{2 i} r_{1 i}, r_{1 i} r_{2 i}\right\}+c_{1 i} c_{2 i}}{2}, c_{1 i} c_{2 i}, \frac{\max \left\{l_{1 i} l_{2 i}, l_{1 i} r_{2 i}, l_{2 i} r_{1 i}, r_{1 i} r_{2 i}\right\}+c_{1 i} c_{2 i}}{2}\right]\right) \\
& =\left[\operatorname{Cov}_{l}\left(\chi_{1}, \chi_{2}\right), \operatorname{Cov}_{c}\left(\chi_{1}, \chi_{2}\right), \operatorname{Cov}_{r}\left(\chi_{1}, \chi_{2}\right)\right]
\end{aligned}
$$




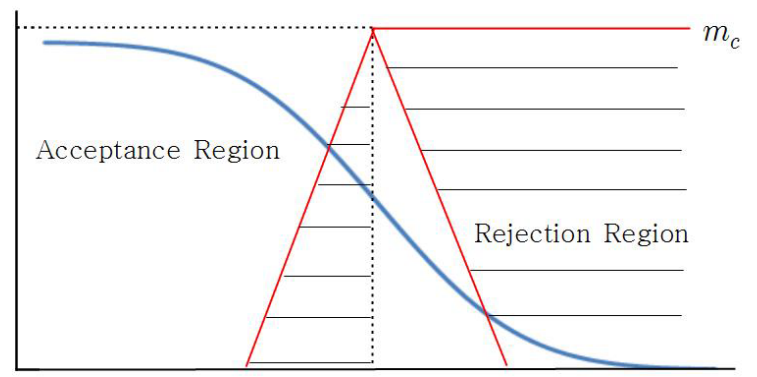

Figure 1: Acceptance and rejection region

by Definition 2 .

Form the reduced variance and covariance, we have fuzzy correlation as;

$$
\widetilde{R_{\chi_{1}, \chi_{2}}}=\operatorname{Cov}\left(\chi_{1}, \chi_{2}\right) \oslash\left(\sqrt{\widetilde{S_{\chi_{1}}^{2}}} \otimes \sqrt{\widetilde{S_{\chi_{2}}^{2}}}\right)=\left[R_{l}, R_{c}, R_{r}\right]
$$

where $\sqrt{\widetilde{S_{k}^{2}}}=\left[\sqrt{\widetilde{S_{i l}^{2}}}, \sqrt{\widetilde{S_{i c}^{2}}}, \sqrt{\widetilde{S_{i r}^{2}}}\right], k=1,2$ for $R_{l} \geq-1$ or $R_{r} \leq 1$.

If $R_{l}<-1$ or $R_{r}>1$ then we have $\left[R_{l}^{\prime}, R_{c}, R_{r}^{\prime}\right]$ from $R_{l}^{\prime}=\left(-1+R_{c}\right) / 2$ or $R_{r}^{\prime}=\left(1+R_{c}\right) / 2$.

\section{Acceptance or Rejection Degree}

Let a test statistic $T$ by fuzzy random sample from sample space $\Omega$. Let $\left\{P_{\theta}, \theta \in \Omega\right\}$ be a family of fuzzy probability distribution, where $\theta$ is a parameter vector of $\Omega$. Choose a membership function $m_{T}(x)$ of $T$ whose value is likely to best reflect the plausibility of the fuzzy hypothesis being tested. Let us consider membership function $m_{C}(x)$ of critical region $C$, which we will call the agreement index of $m_{T}(x)$ which regard to $m_{C}(x)$.

Definition 3. Let a fuzzy membership function $m_{T}(x), x \in \mathfrak{R}$, we consider another membership function $m_{C}(x), x \in \mathfrak{R}$, which call the agreement index, the ratio being defined in the following way by Kang et al. (2003)

$$
m_{A G}(x)=\frac{\operatorname{area}\left(m_{T}(x) \cap m_{C}(x)\right)}{\operatorname{area}\left(m_{C}(x)\right)} \in[0,1]
$$

Definition 4. We define real-valued function $\mathfrak{J}^{(\delta)}$ by supermum grade of membership function for rejection or acceptance degree by an agreement index of $\delta$-level by Kang et al. (2003) as

$$
\begin{aligned}
& \mathfrak{J}^{(\delta)}(0)=\sup _{\delta}\left\{\frac{\operatorname{area}\left(m_{T^{(\delta)}}(\theta) \cap m_{C^{(\delta)}}(\theta)\right)}{\operatorname{area}\left(m_{C^{(\delta)}}(\theta)\right)}\right\}, \\
& \mathfrak{J}^{(\delta)}(1)=1-\mathfrak{J}^{(\delta)}(0)
\end{aligned}
$$

for the fuzzy hypothesis testing, respectively.

We show the acceptance region and rejection region for the fuzzy critical region $C$ as Figure 1 . For various kinds of $T$, we can reject the hypotheses by Definition 4 as Figure 2. 


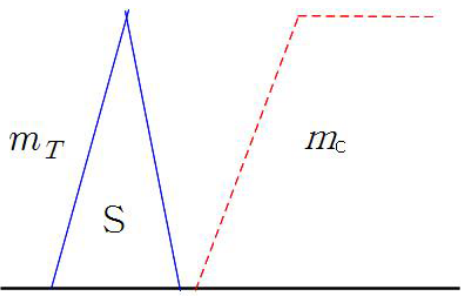

(a)

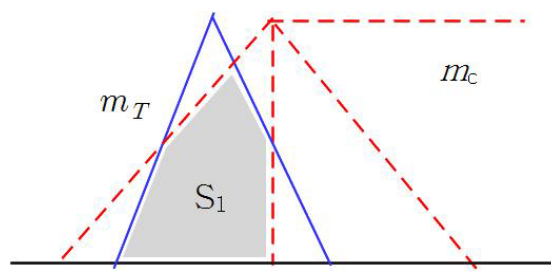

(c)

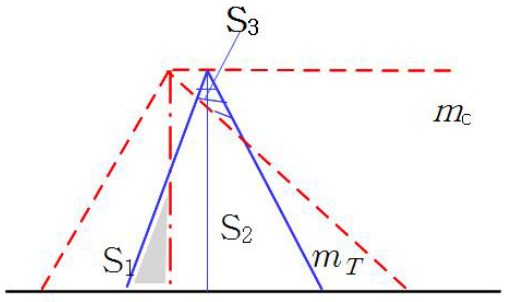

(e)

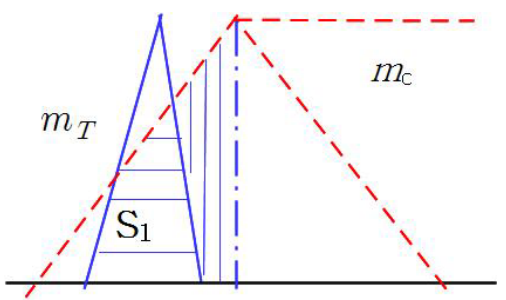

(b)

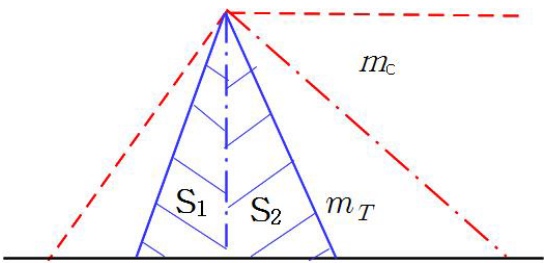

(d)

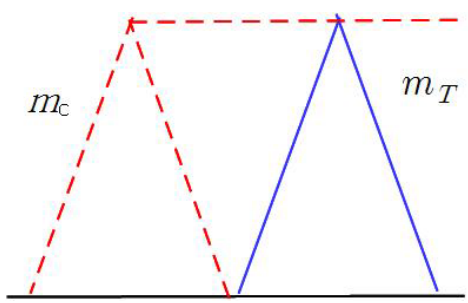

(f)

Figure 2: Various type of $m_{C}$ by $m_{T}$

For example in (a) or (f) of Figure 2, we can clearly reject the hypothesis as degree $\mathfrak{J}^{(\delta)}(0)=0$ or $\mathfrak{J}^{(\delta)}(0)=1$, respectively.

For (b) of Figure 2, we have

$$
\mathfrak{J}^{(\delta)}(0)=\frac{\operatorname{area}\left(s_{1}\right)+\operatorname{area}\left(s_{2}\right)}{\operatorname{area}\left(s_{1}\right)+\operatorname{area}\left(s_{2}\right)+\operatorname{area}\left(s_{3}\right)} \times \frac{1}{2}
$$

for left hand side of triangular fuzzy critical region $m_{C}$ by fuzzy the statistics $m_{T}$ where $1 / 2$ is a weighted value for $-1 \leq \mathfrak{J}^{(\delta)}(0), \mathfrak{J}^{(\delta)}(1) \leq 1$.

In case (d) of Figure 2, we have

$$
\mathfrak{J}^{(\delta)}(0)=\frac{\operatorname{area}\left(s_{1}\right)+\operatorname{area}\left(s_{2}\right)}{\operatorname{area}\left(s_{1}\right)+\operatorname{area}\left(s_{2}\right)} \times \frac{1}{2}=0.5,
$$

it maintains an uncertain attitude for decision in the hypotheses.

In addition, we have

$$
\mathfrak{J}^{(\delta)}(0)=\frac{\operatorname{area}\left(s_{1}\right)+\operatorname{area}\left(s_{2}\right)}{\operatorname{area}\left(s_{1}\right)+\operatorname{area}\left(s_{2}\right)} \times \frac{1}{2}+\frac{\operatorname{area}\left(s_{4}\right)+\operatorname{area}\left(s_{5}\right)}{\operatorname{area}\left(s_{4}\right)+\operatorname{area}\left(s_{5}\right)+\operatorname{area}\left(s_{6}\right)} \times \frac{1}{2}
$$

in (e) of Figure 2. 


\section{Power-Normal Fuzzy Distribution}

From non-normal random fuzzy sample $o(\chi)$ by $\delta=0$, if we apply an inverse transformation by the Box-Cox power transformation then we have a normal random fuzzy variable $\chi$ by Goto and Hamasaki (2002) as;

$$
\chi= \begin{cases}(\lambda \cdot o(\boldsymbol{\chi})+1)^{\frac{1}{\lambda}}, & \text { for } \lambda \neq 0 \\ \exp (o(\boldsymbol{\chi})), & \text { for } \lambda=0 .\end{cases}
$$

The Equation (5.1) have a power-normal fuzzy distribution $(\mathrm{PN})$ as $\operatorname{PN}\left(\lambda, \tilde{\mu}, \widetilde{\sigma^{2}}\right)$. Since $\boldsymbol{\chi} \sim$ $\operatorname{PN}\left(\lambda, \tilde{\mu}, \widetilde{\sigma^{2}}\right)$, for the power-normal random fuzzy variable $\chi$, the probability distribution function by Freeman and Modarres (2001)

$$
f\left(\chi \mid \lambda, \tilde{\mu}, \widetilde{\sigma^{2}}\right)=\frac{1}{K} \frac{1}{\sqrt{2 \pi} \tilde{\sigma}} \chi^{\lambda-1} \exp \left[-\frac{1}{2}\left(\frac{p \lambda(\chi)-\tilde{\mu}}{\tilde{\sigma}}\right)^{2}\right]
$$

is power-normal fuzzy distribution, where $\chi^{\lambda-1}$ is Jacobian.

We consider some properties of fuzzy $t$-test statistics for testing independence of bivariate nonnormal distribution obtained from the random fuzzy samples.

Since $\sqrt{n-2} \rho / \sqrt{1-\rho^{2}}$ has $t$-distribution with $n-2$ degree of freedom in Anderson (1971). Against alternatives $R_{\chi_{1}, \chi_{2}} \leq C_{0}$, we reject $H_{f 0}: \rho \leq \theta_{0}$ if

$$
\frac{R_{\chi_{1}, \chi_{2}}}{\sqrt{\left(1-R_{\chi_{1}, \chi_{2}}^{2}\right) /(n-2)}}>t_{n-2}(\alpha) .
$$

For testing the independence of bivariate normal fuzzy distribution, we have sample fuzzy correlation by transformed power normal distribution between $\chi_{1}$ and $\chi_{2}$ as $\widetilde{R_{\chi_{1}, \chi_{2}}}=\operatorname{Cov}\left(\chi_{1}, \chi_{2}\right) /\left(\sqrt{\widetilde{S_{\chi_{1}}^{2}}} \sqrt{\widetilde{S_{\chi_{2}}^{2}}}\right)$ from Equation (3.8).

The fuzzy hypothesis $H_{f 0}: m_{\rho} \leq m_{\theta_{0}}$ is a equivalent to $m_{R_{\chi_{1} x_{2}}} \leq m_{c_{0}}$ where $\rho$ is population correlation and $\theta_{0}$ is a parameter.

It follows from the bivariate normal distribution for fuzzy testing fuzzy hypothesis $H_{f 0}$ and we reject when

$$
\widetilde{R_{\chi_{1}, \chi_{2}}}>\widetilde{C_{0}}
$$

or equivalently when

$$
\frac{\widetilde{R_{\chi_{1}, \chi_{2}}}}{\sqrt{\left(1-\widetilde{R_{\chi_{1}, \chi_{2}}^{2}}\right) /(n-2)}}>\widetilde{K_{0}},
$$

where $\widetilde{C}_{0}$ and $\widetilde{K}_{0}$ are a fuzzy number by fuzzy significance levels for $\delta=0$.

The fuzzy test of the fuzzy hypothesis $H_{f 0}: m_{\rho}=\tilde{0}$ against the alternatives not $\rho$ is near to , therefore rejects when

$$
\frac{\left|\widetilde{R_{\chi_{1}, \chi_{2}}}\right|}{\sqrt{\left(1-\widetilde{R_{\chi_{1}, \chi_{2}}^{2}}\right) /(n-2)}}>\widetilde{K_{1}}
$$

for any fuzzy number $\widetilde{K}_{1}$ by fuzzy significance levels. 
Table 1: Blood pressure sample

\begin{tabular}{|c|c|}
\hline$\overline{o(X) \text { (systolic) }}$ & $o(Y)$ (diastolic) \\
\hline$[114,116,120]$ & {$[68,70,73]$} \\
\hline$[91,94,98]$ & {$[67,68,72]$} \\
\hline$[114,120,127]$ & {$[82,86,90]$} \\
\hline$[124,130,138]$ & {$[74,80,88]$} \\
\hline$[123,134,139]$ & {$[76,82,86]$} \\
\hline$[113,119,124]$ & {$[76,79,84]$} \\
\hline$[123,128,135]$ & {$[76,82,85]$} \\
\hline$[104,112,117]$ & {$[74,80,83]$} \\
\hline$[102,110,122]$ & {$[78,81,84]$} \\
\hline$[98,103,109]$ & {$[77,85,89]$} \\
\hline$[123,134,137]$ & {$[82,87,92]$} \\
\hline$[102,110,122]$ & {$[82,84,87]$} \\
\hline$[135,141,145]$ & {$[75,82,85]$} \\
\hline$[114,120,125]$ & {$[68,73,75]$} \\
\hline$[109,112,116]$ & {$[72,76,80]$} \\
\hline
\end{tabular}

\section{Illustration}

Since $\sqrt{n-2} R_{X, Y} / \sqrt{1-R_{X, Y}^{2}}$ has the $t$-distribution with $n-2$ degree of freedom when $\rho=0$, the constants $K_{0}$ and $K_{1}$ in the above tests are given by

$$
\int_{K_{0}}^{\infty} t_{n-2}(x) d x=\alpha, \quad \int_{K_{1}}^{\infty} t_{n-2}(x) d x=\frac{\alpha}{2}
$$

by significance level $\alpha$.

The distribution $R_{X, Y}$ depends only on the correlation coefficient $\rho$, also it is true of the power of these tests.

From a bivariate probability distribution of blood pressures sample by Table 1, we induce fuzzy variance and covariance by transformed power-normal data for $\lambda=1 / 2$ and $\delta=0$ from Equation (5.1), (3.4) and (3.5) as $\widetilde{R_{X, Y}}=[-0.33,0.43,0.72]$.

From Equation (3.8), we have $t$-statistics for $\delta=0$ as;

$$
\frac{\left|\widetilde{R_{X, Y}}\right|}{\sqrt{\left(1-\widetilde{R_{X, Y}^{2}}\right) /(n-2)}}=[1.26,1.73,3.70] \text {. }
$$

For $H_{f 0}: m_{\rho}=\tilde{0}$, if we have fuzzy significance level $\widetilde{\alpha}=[0.025,0.05,0.75]$ and $d . f .=13$ then $\widetilde{K_{1}}=[1.94,2.16,2.53]$ by Equation $(5.7)$.

Since $[1.26,1.73,3.70]>\widetilde{K_{1}}$, the degree of acceptance for the hypothesis is $\mathfrak{J}^{(\delta=0)}(1)=0.92$ by Equation (3.2) and Equation (4.6) as Figure 3.

\section{Remarks and Conclusion}

We would like to show the reduced-spread fuzzy variance and covariance for them average with respect to the spreads by a fuzzy number center and agreement index by area ratio for acceptance or the rejection degree of the fuzzy hypotheses.

We tested the independence of bivariate normal distribution by Box-Cox power fuzzy transformation for non-normal data blood pressure samples by an agreement index. 


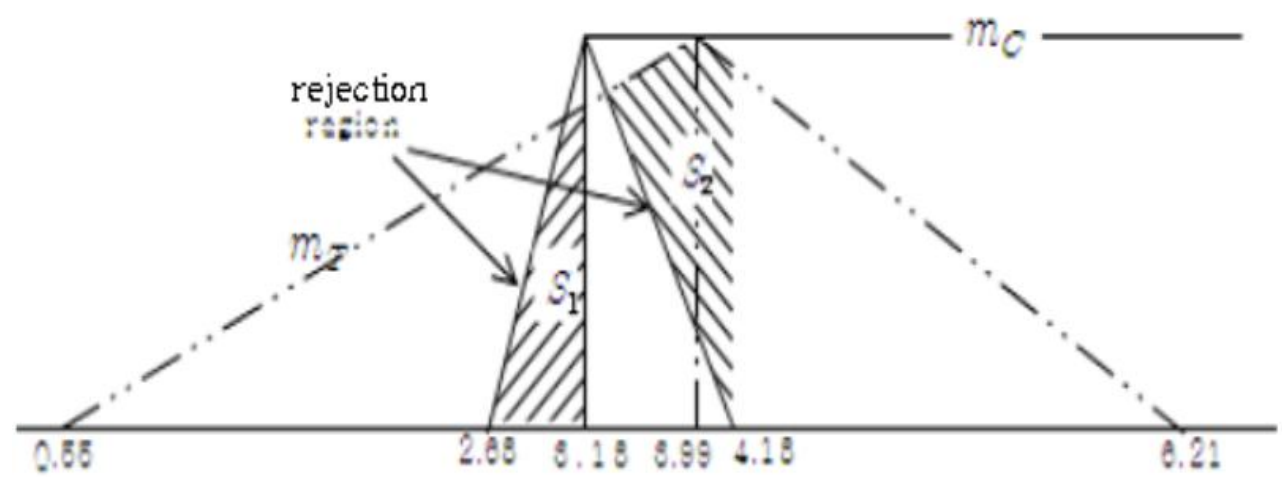

Figure 3: Fuzzy critical region $m_{C}$ by test statistics $m_{T}$

For crisp data by $\delta=1$, we accept the hypothesis $H_{0}: \rho=0$ with acceptance degree $\mathfrak{J}^{(\delta=1)}(0)=$ 1.0 by

$$
\frac{\widetilde{R_{\chi_{1}, \chi_{2}}}}{\sqrt{\left(1-\widetilde{R_{\chi_{1}, \chi_{2}}^{2}}\right) /(n-2)}}=[1.73,1.73,1.73]<t_{15-2}(\widetilde{0.025})=[2.16,2.16,2.16] .
$$

For $\delta=1$, we accept the hypothesis $H_{f 0}: m_{\rho}=\tilde{0}$ by acceptance degree $\mathfrak{J}^{(\delta=0)}(0)=0.92$ within the range of possibility; however, we will accept the alternative hypothesis by rejection degree $\mathfrak{J}^{(\delta=0)}(1)=0.08$.

Thus we have very useful and flexible testing hypotheses concerning the fuzzy distributions for uncertainty data.

\section{References}

Anderson, T. W. (1971). Multivariate Statistical Analysis, John Willy \& Sons.

Colubi, A. (2009). Statistical inference about the means of fuzzy random variables: Applications to the analysis of fuzzy-and real-valued data, Fuzzy Sets and Systems, 160, 344-356.

Freeman, J. and Modarres, R. (2001). Properties of the power-normal distribution, Analysis of Transformed Environmental Data with Detection Limits, Report for 2001 DC3921B.

Gizegorzewski, P. X. (2000). Testing hypotheses with vague data, Fuzzy Sets and Systems, 112, 501510.

Goto, M. and Hamasaki, T. (2002). The bivariate power-normal distribution, Research Association of Statistical Science, 34, 29-49.

Kang, M. K., Lee, C. E. and Han, S. I. (2003). Fuzzy hypotheses testing for hybrid numbers by agreement index, Far East Journal of Theoretical Statistics, 10, 1-9.

Kang, M. K. and Seo, H. A. (2009). Fuzzy hypothesis test by Poisson test for most powerful test, Journal of Korean Institute of Intelligent Systems, 19, 809-813.

Watanabe, N. and Imaizumi, T. (1993). A fuzzy statistical test of fuzzy hypotheses, Fuzzy Sets and Systems, 53, 167-178. 